\title{
SURVEY ON LI-Fi TECHNOLOGY AND ITS APPLICATIONS
}

\author{
Hema Patel \\ Assistant Professor, Smt. Chandaben Mohanbhai Patel Institute of Computer Applications, \\ CHARUSAT, Changa
}

\begin{abstract}
This paper describes the importance of the Li-Fi technology and the areas where it can be advantageous. Li-Fi can prove to be a boon to our society as it saves not only money and resources of user butat the same time also enables the user to take the advantage of high data rate transmission that is about 1000 times more efficient than existing Wi-Fi data transfer technology that uses radio frequency. This paper also emphasizes how it becomes possible to use a light source as transmitter of data.
\end{abstract}

\section{Keywords}

Li-Fi (Light Fidelity), RF (Radio Frequency), LED (Light Emitting Diode).

\section{INTRODUCTION}

Today, we have many tools and technology for communication. To communicate wired as well as wireless technology available. Li-Fi is one of the emerging wireless technologies among them.

The Light-Fidelity came into existence in year 2011 Coined by a team of scientist Dr. Gordon Povey, Prof. Harald Haas and Dr. Mostafa Afgani at Edinburgh University in U.K. They began their research in this field in 2004. On 12th July 2011, the original founder Prof. Harald Hass discussed about his invention and coined the term Li-Fi at the TED Global conference in Edinburgh.

Li-Fi is a kind of optical wireless communications (OWC) and can be used as a complement to RF communication (Wi-Fi or Cellular network), or a replacement in contexts of data broadcasting. That is currently attracting a great deal of interest as it offers an opportunity to its users to enjoy high data transfer rate at low cost, that is required as a growing number of people and their recent devices access wireless internet, the waves in the air are becoming increasingly clogged making it more and more difficult to get the signals that are reliable and high in speed [1].

Light is in fact very much part of our lives for millions and millions of years. So, Li-Fi is present wherever there is availability of light. So by using the light to transmit the data we can in turn demolish the necessity of having hot-spots. This is the opportunity to exploit a different part of the electromagnetic spectrum that is still not greatly utilized.

Li-Fi has other advantages over Wi-Fi, such as usage of Li-Fi technology does not harm nuclear power plants, thermal power stations where Wi-Fi cannot be used because in such stations usage 
International Journal of Information Sciences and Techniques (IJIST) Vol.6, No.1/2, March 2016 of RF waves can be harmful and may result in unexpected accident.

So, there are different criterions on the basis of which we can judge the working of Li-Fi and WiFi that is efficiency, capacity, availability and security [1].

\section{How LI-FI WORKS?}

In Li-Fi, data transmits through LEDs thus the area of the screen which illuminates light can be served as a platform for data communication. Figure 1.1 shows the conversion of light into digital data.

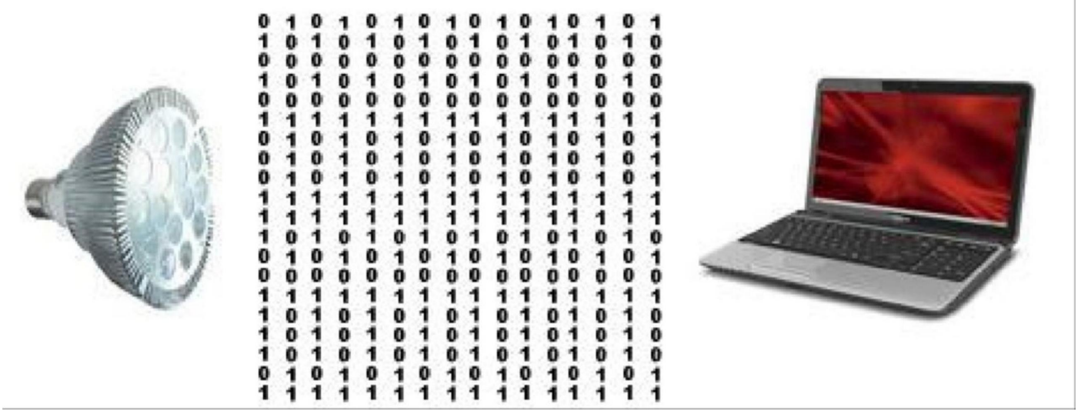

Figure 1 Basic Principle of Li-Fi Technology

To implement Li-Fi developers have used white LED light bulbs. To make Li-Fi works, constant current is required. The developers decided to use LED light bulb as it is a semi-conductor device. It can blink rapidly so development of binary signals becomes possible. The light intensity can be manipulated to send data by tiny changes in amplitude. The benefit of using LED is that the LEDs also provide light as any incandescent bulb. This property of optical current is used in Li-Fi setup.

Flickering is possible in the LED light. LED lights can flicker thousands of times every second and by altering the amount of the flickers we can produce the series of zero and one to introduce digital communications. If the LED bulb is on, a digital 1 is transmitted and if it is off a 0 is transmitted. So, to make Li-Fi work, we require some LEDs and a controller that can code data into those LEDs and change the rate at which the LEDs can flicker. The LED intensity is modulated so rapidly that human eyes cannot notice, so the output appears constant [2].

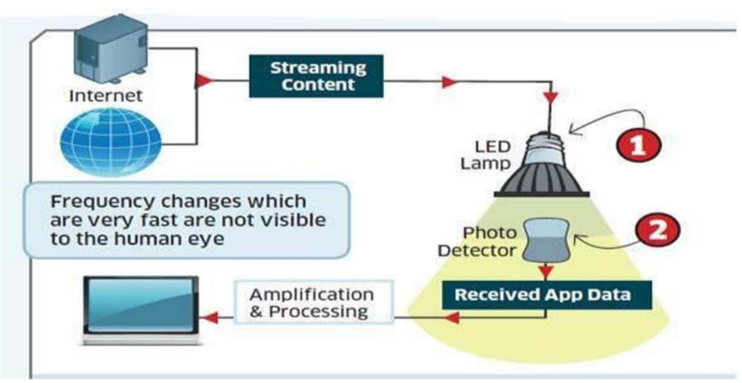

Figure 2 Li-Fi Review [2] 


\section{OUTLOOK OF LI-FI}

The screen of the mobile phone, monitor, television, bulbs can act as a source of light. On the other hand, the receiving platform, camera of mobile phone can be used as photo detector to scan and retrieve data.

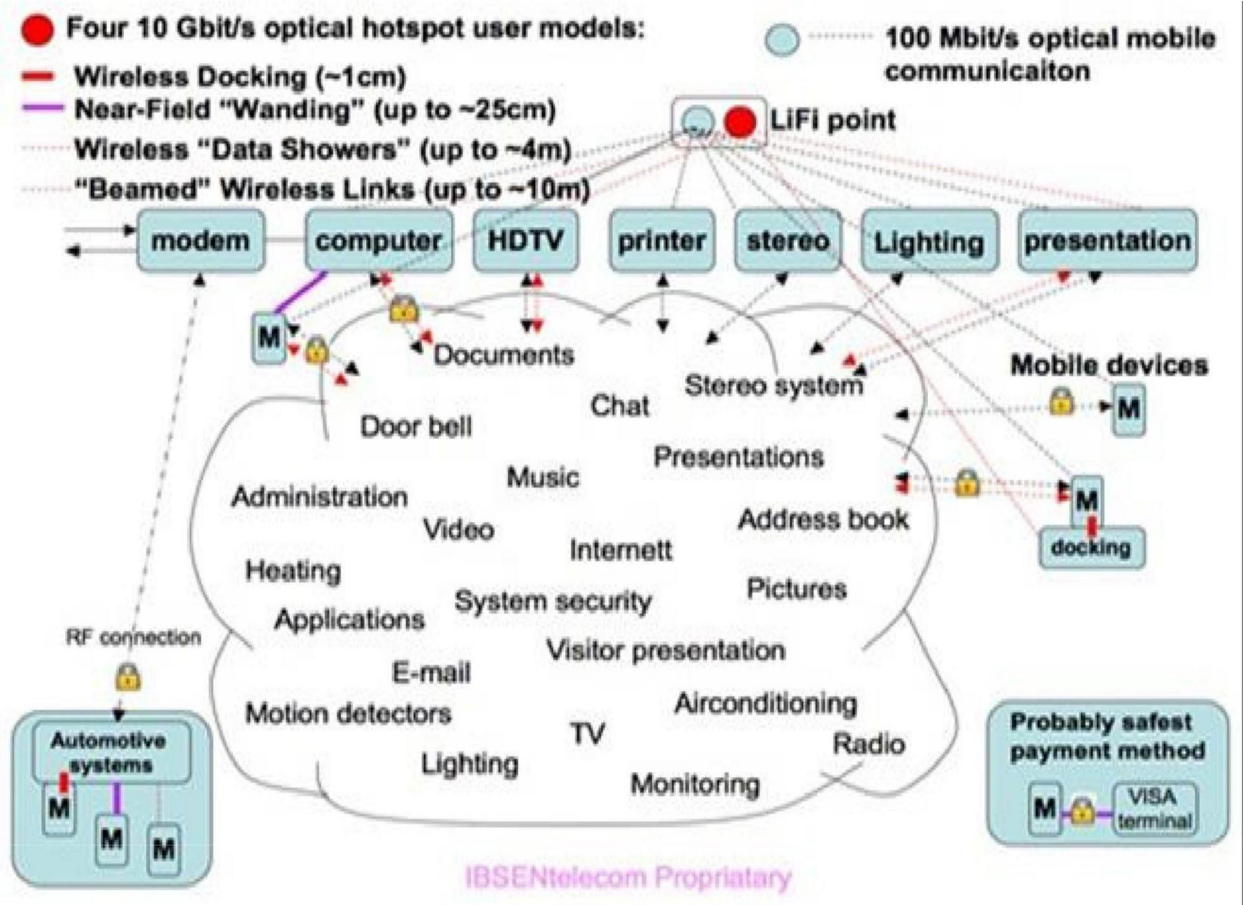

Figure 3 Li-Fi Communications with other devices

In figure 1.3 shows how the Li-Fi cloud communicates with others devices. Li-fi is using visible light instead of radio waves for communication. In the case of Li-Fi there is no need to use complex network of wires and box which is installed in the case of Wi-Fi. Li-Fi translates the light pulses off or on in classic binary language of zeros and ones through tiny LED bulbs on and off a million of times per second. The flickering of LEDs can allow user to access wireless internet with data transmission at speeds of near to $10 \mathrm{Gbit} / \mathrm{s}$, theoretically. So the user may download a high-definition film within 30 seconds which is 250 times faster than superfast broadband [1].

\section{APPlications OF Li-Fi}

Here, we will explore many ways in which we can put our Li-Fi to better use including directional lighting, energy efficiency, intrinsic security, high data rate capability, signal blocking by walls and integrated networking capability[3][4][5]. 
International Journal of Information Sciences and Techniques (IJIST) Vol.6, No.1/2, March 2016 Each artificial light source in the application environment becomes a separate data channel. These channels can supply different data into each separate pool of light, delivered at the full rated download speed for that channel.

\subsection{Security}

In a conference room, the access area of each channel depends on the width of the light pool, and at the same time can be accessed by multiple users present within that environment. When we are using Li-Fi, each user present in the application environment can receive higher data rates than would be the case with an equivalent Wi-Fi channel. In the case of Wi-Fi, each user or group of users directly competes for access to bandwidth that slower the download speeds as more connections are there. By contrast, in the case of Li-Fi that provides greater number of available access points, each source of light provides full channel data rates with fewer simultaneous users. So, Li-Fi provides benefit of 1000 times greater speed to each user. In addition, and in contrast to radio waves, the light does not pass through the walls. Therefore, with minimal precautions to avoid leakage from windows, doors, etc., security is fundamentally enhanced as compared with Wi-Fi.

\subsection{Dense Urban Environments (Interference-free Wireless Communication)}

Dense urban environments cover larger area with artificial lighting. The area with lighting infrastructure can provide high data rate access for users as the users move through that environment. For example, in a hotel corridor or reception hall a number of users can receive high data rate at any point. Moreover, high speed wireless communication would be available in every room since the light waves cannot propagate through walls. This results in interference-free wireless communication.

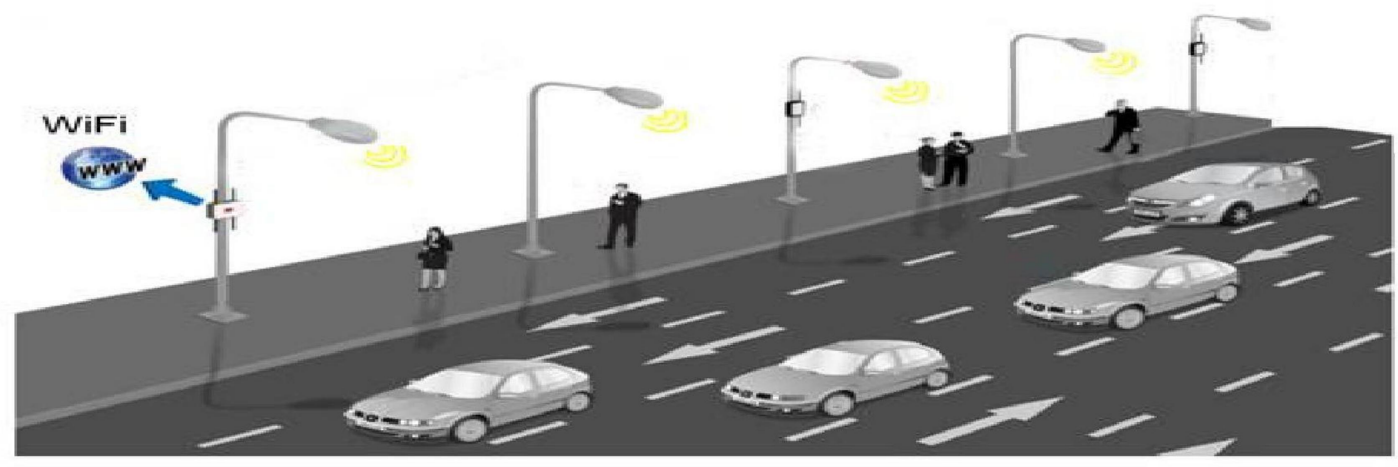

Figure 4 Smart Urban Communication Network

Other employments of Li-Fi innovation in outside urban territory is that Li-Fi can empower road lights that would give a system of access focuses for web. In cell correspondence, the separation between radio base stations is less. In this way, rather than conveying new radio base stations in our urban areas, road lights could give both, light amid night and information correspondence at 
International Journal of Information Sciences and Techniques (IJIST) Vol.6, No.1/2, March 2016 rapid every minute of every day. In this way, expense of arrangement and establishment of new radio base station can be spared. Shockingly, notwithstanding when the lights are off as saw by the eye, high information correspondence rates are still conceivable.

\subsection{EMI Sensitive Environments}

Use of Li-Fi in aircraft, allow passenger to take the advantage of high data rate connectivity at all time without generating electromagnetic interference (EMI) interference (EMI) with sensitive radio equipment on the flight deck. The reduction in cabling requirement also means a lighter aircraft.

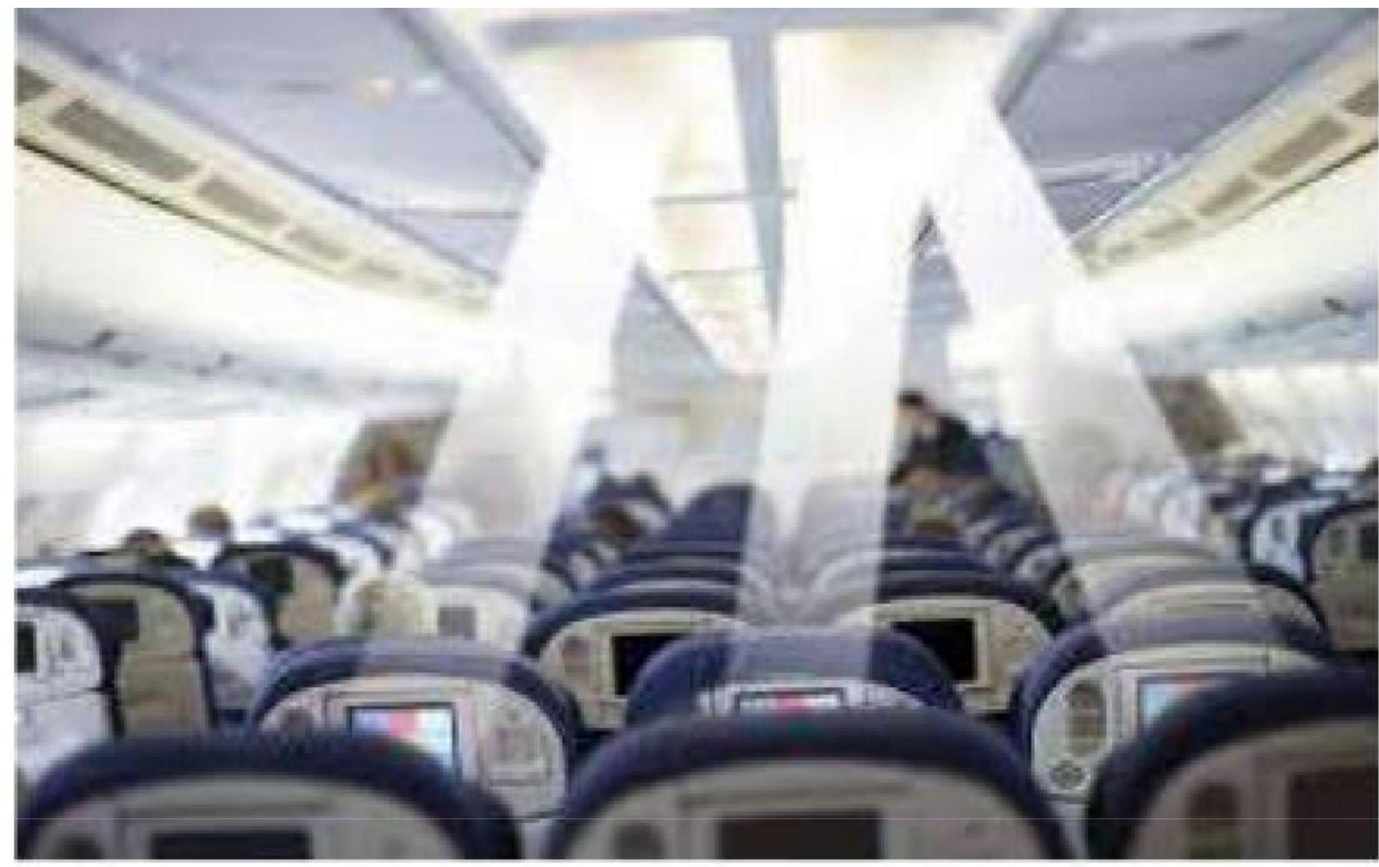

Figure 5 Communications in Airlines [6]

\subsection{Augmented Reality}

Exhibitions in museums and galleries are illuminated with artificial lighting. Li-Fi technology enables lighting can provide localized information. So that a visitor $\mathrm{s}$ camera or mobile phone can be used to download further information regarding the object being viewed from the light.

\subsection{Localized Advertising}

By merging street lights and lighting display means of a shop as a Li-Fi broadcast channel, it is possible to transmit advertising information like special offers and coupons. So, this provides opportunity to emerge novel retail business model. Product information, catalogue information, discount coupons and advertising videos could all be provided to shoppers. 


\subsection{Underwater Communication}

In the case of Wi-Fi, it is not possible to communicate through water as radio waves are quickly absorbed in water that prevents underwater radio communications, but light can penetrate for large distances. Therefore, Li-Fi can enable underwater communication.

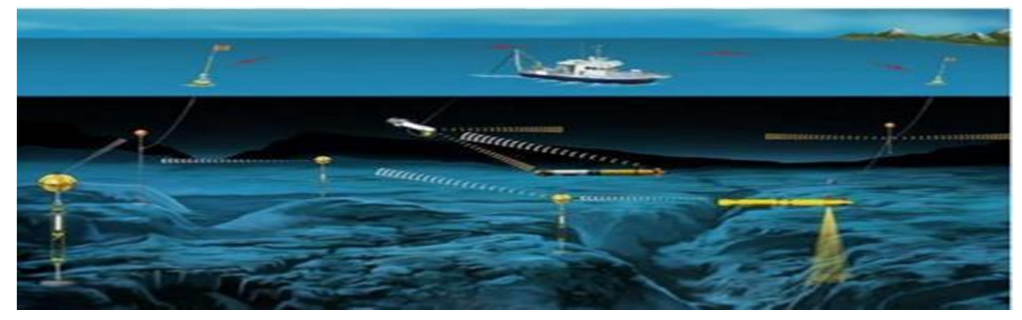

Figure 6 Communication under water [6]

\subsection{Safety Environments}

The use of electrical equipment like mobile phones in explosion hazard environments is generally restricted. With the use of Li-Fi technology to pass data will simplify the configuration of data networks and can enable new systems to enhance security in these environments.

\subsection{Intelligent Transportation Systems}

Now-a-days headlights and tail lights of car are steadily being replaced with LED versions. This offers the possibility of car-to-car communication using Li-Fi, allowing development of anticollision systems and exchange of information about driving conditions between vehicles. Traffic lights can also be used that already use LED lighting, so it provides the opportunity to manage traffic systems. This would also enable car systems to download information available on the network regarding optimal routes to take, and update the network regarding conditions recently experienced by individual vehicles.

\subsection{Connectivity}

Our homes as of now have lighting broadly introduced. The utilization of Li-Fi empowered lighting will change the applications that can be conceived, not just the interconnection of gadgets, for example, TVs,

PCs and Hi-Fi, additionally interfacing conventional local apparatuses, for example, ice chests, clothes washers, microwaves and vacuums. The "web of everything". 


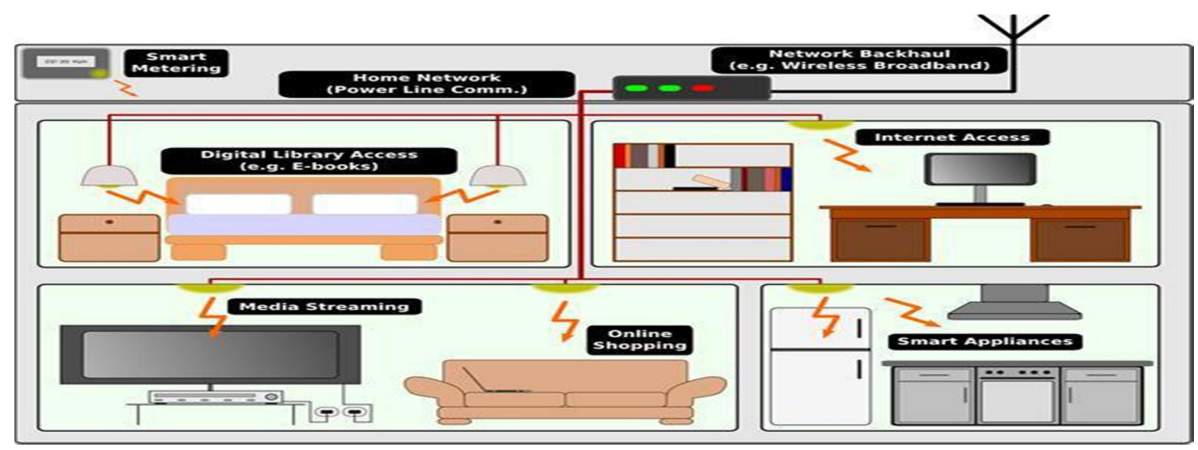

\subsection{Sensitive Data}

Figure 7 Li-Fi Communications at Home [7]

Every hospital has large amount of data and a huge collection of equipments that may cause electromagnetic interferences (EMI). So, in this environment both EMI sensitivity and security of data are issues. The use of Li-Fi is helpful that can enable the better deployment of more secured network of medical instruments, patient records, etc.

\subsection{Indoor Navigation}

By recognizing every light (for instance, through the utilization of the generally utilized MAC codes utilized by information switches and PCs) it is conceivable to give a shrewd method for exploring through urban situations. The ID of every code would be connected to a particular area. For instance, light got from the nearest apparatus can demonstrate to a versatile client their careful position as they go along a passage.

\section{CONCLUSION}

The concept of Li-Fi is currently attracting a great deal of interest, not least because it may offer a genuine and very efficient alternative to radio-based wireless. So, this paper concludes that Li-Fi is great for high density wireless data coverage in comparatively smaller area (covered by a LED bulb) and to sort out issues rose due to radio frequency. If we implement Li-Fi technology, each and every bulb can be used as a Wi-Fi hotspot through which we can transmit data to multiple users at high speed and it becomes possible to keep our environment clean, green and safe. Although Li-Fi has various advantages but it cannot be thought as a replacement of technology uses radio frequency as light using in this technology cannot be passed through obstacles.

\section{ACKNOWLEDGements}

This paper is made possible through the help and support from everyone, including: parents and colleague. Especially, please allow me to dedicate my acknowledgment of gratitude toward the following significant advisors and contributors:

First and foremost, I would like to thank our Principal Dr. Atul Patel for his most support and encouragement.

Second, I would like to thank Mr. Kanubhai K. Patel and Ms. Hetal Patel who offered invaluable detailed advices on organization and the theme of the paper. 
International Journal of Information Sciences and Techniques (IJIST) Vol.6, No.1/2, March 2016

\section{REFERENCES}

[1] revathi ganesan, (2014) li-fi technology in wireless communication , international journal \& magazine of engineering, technology, management and research, issn no: 2348-4845.

[2] http://www.lifi-lab.com/lifi/lifi-enhanced-mode-future-wireless-communication-review.html

[3] http://www.lifi-centre.com/about-li-fi/applications/.

[4] warudkar, r. S., \& malani, s. S. Li-fi: a new approach in wireless communication.

[5] mihir chauhan and aditya kulai (2015), li-fi let there be light , international journal of engineering trends and technology (ijett) volume 28.

[6] http://www.slideshare.net/uzmaruhy/lifi-perfect-slides.

[7] http://www.configurarequipos.com/usuario-jesusgrob/li-fi-llegara-primero-a-la-republica-mexicana

\section{Author}

Hema Patel has completed her Bachelor of Computer Applications from Vikram University, Ujjain, in year 2006. In year 2009, she received degree of Master of Computer Applications from Rajiv Gandhi Technical University, Bhopal. Currently, She is Assistant Professor at Smt. Chandaben Mohanbhai Patel Institute of Computer Applications, CHARUSAT University of Science and Technology, situated at Changa, Gujarat.

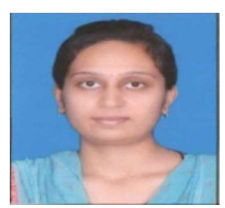

